\title{
Adsorção de fenol e benzeno em montmorilonita modificada com brometo de hexadeciltrimetilamônio
}

\section{(Adsorption of phenol and benzene in montmorillonite modified with hexadecyltrimethylammonium bromide)}

\author{
S. S. dos Santos, V. P. Lemos, A. L. C. Vilaça, L. Tavares, L. S. Queiróz \\ Instituto de Geociências, Universidade Federal do Pará, Av. Augusto Corrêa s/n, Belém, PA 66075-110 \\ sheilasantos_ufpa@yahoo.com.br
}

\begin{abstract}
Resumo
Este estudo avalia a intercalação de esmectita dioctahédrica do Estado Acre com o sal quaternário de amônio brometo de hexadeciltrimetilamônio (HDTMA) em concentrações compreendidas entre 1 a 5 vezes ao valor de CTC da argila original e sua aplicabilidade na adsorção de fenol e benzeno em fase líquida. Os materiais foram caracterizados por microanálises de difração de raios X, microscopia eletrônica de varredura/espectroscopia com dispersão de energia e espectroscopia na região do infravermelho. Os processos de adsorção foram realizados em sistemas simples e as medidas das concentrações do fenol e benzeno foram efetuadas por espectrofotometria na região ultravioleta. Os resultados indicaram que a intercalação da esmectita com HDTMA propiciou cerca de 78,8\% de expansão (variação de $\mathrm{d}_{001}: 1,47 \mathrm{~nm}$ para 1,91 nm) e decréscimo acentuado dos elementos das posições interlamelares, como $\mathrm{Na}, \mathrm{Ca}, \mathrm{Mg}$ e K, acompanhado pela delaminação/esfoliação da argila original. Nos processos de adsorção do fenol e benzeno em HDTMA-arg 5 foram obtidos os seguintes resultados: $\mathrm{q}_{\max }=4,44$ e $35,8 \mathrm{mg} \cdot \mathrm{g}^{-1} ; \mathrm{K}_{\mathrm{L}}=0,076 \mathrm{e}$ e,115 L.g $\mathrm{g}^{-1} ; \Delta \mathrm{G}^{\mathrm{o}}=-16,85$ e $-18,38 \mathrm{~kJ}_{\mathrm{mol}}{ }^{-1}$, respectivamente. Estes dados indicaram que os processos de adsorção do benzeno e fenol em fase líquida na organoargila HDTMA-arg 5 foram favoráveis, espontâneos e com interações físicas.
\end{abstract}

Palavras-chave: esmectita, organoargila, adsorção, fenol, benzeno.

\begin{abstract}
This study evaluates the intercalation of the smectite dioctahedric state of Acre, Brazil, with the quaternary ammonium salt bromide, hexadecyltrimethylammonium (HDTMA) at concentrations ranging from 1 to 5 times the value of the original clay CEC and its application in the adsorption of phenol and benzene in the liquid phase. The materials were characterized by microanalysis X-ray diffraction, scanning electron microscopy/energy dispersive spectroscopy and infrared spectroscopy. The adsorption processes were carried out in simple systems and measurements of the concentrations of phenol and benzene were performed by spectrophotometry in the ultraviolet region. The results indicated that the intercalation of smectite with HDTMA provided approximately 78.8 expansion (range $d_{001}: 1.47 \mathrm{~nm}$ to $1.91 \mathrm{~nm}$ ) and sharp decrease of the interlayer positions of elements such as $\mathrm{Na}, \mathrm{Ca}, \mathrm{Mg}$ and $\mathrm{K}$, together by delamination/exfoliation of the clay original. In adsorption processes of phenol and benzene at HDTMA-arg 5 the following results were obtained: $q_{\max }=4.44$ and $35.8 \mathrm{mg} \cdot \mathrm{g}^{-1} ; K_{L}=0.076$ and $0.115 \mathrm{~L}_{\mathrm{g}} \mathrm{g}^{-1} ; \Delta \mathrm{G}^{o}=-16.85$ and $-18.38 \mathrm{~kJ}^{\mathrm{mol}} \mathrm{mol}^{-1}$, respectively. These data indicated that the adsorption of benzene and phenol in liquid phase in the organophilic HDTMA-arg 5 were favorable, spontaneous and physical interactions.
\end{abstract}

Keywords: smectite, organoclay, adsorption, phenol, benzene.

\section{INTRODUÇÃ̃O}

Os minerais de argila, como esmectitas, são muito utilizadas nas suas formas naturais como adsorventes de íons metálicos, pois apresentam cristais com pequenas dimensões, elevada capacidade de troca catiônica (CTC) e facilidade em aumentar a distância interplanar basal $\mathrm{d}_{001}$ fazendo com que as reações de intercalação sejam mais rápidas [1]. Na sua forma natural, a montmorilonita possui carga negativa na superfície que pode ser substituída isomorficamente. Ela é, naturalmente, balanceada pelos cátions inorgânicos trocáveis, tais como $\mathrm{Na}^{+} \mathrm{e} \mathrm{Ca}^{2+}$, que são geralmente hidratados. Quando as posições interlamelares da montmorilonita são intercaladas com cátions de sais quaternários de amônio (com pelo menos 12 ou mais átomos de carbono em sua cadeia), sua natureza hidrofílica transforma-se em hidrofóbica, obtendo-se neste processo organoargilas $[2,3]$.

Os surfactantes catiônicos em suspensões aquosas de minerais de argila expansíveis, como os do grupo da esmectita, deslocam os cátions trocáveis dos espaços interlamelares da argila e o arranjo dos cátions dos surfactantes nesses espaços depende da carga e tamanho da cadeia carbônica e quantidades adsorvidas dos surfactantes 
[3]. Se o surfactante é adicionado em quantidade superior a CTC da argila, então o excesso é adsorvido via interação Van der Waals [4-6].

Entre as várias aplicações industriais e tecnológicas das organoargilas constam o uso em polímeros, materiais elétricos, nanocompositos [7], como barreira de adsorção de contaminantes tóxicos orgânicos e inorgânicos $[8,9]$. Atenção especial tem sido dada nas últimas décadas sobre adsorção de compostos orgânicos, como fenóis que são potenciais contaminantes do lençol freático [10-12]. Outros poluentes de grande preocupação global são os compostos orgânicos voláteis, como benzeno [13-15]. Esses compostos provocam danos à saúde, principalmente devido à toxicidade e/ou mutagenicidade ou carcinogenicidade. Dentre os cânceres, as leucemias são as mais freqüentes e, dentre elas, as mais comuns, são as agudas. No Brasil, a ação cancerígena do benzeno foi reconhecida oficialmente a partir de 1994, pela portaria da secretaria de segurança e saúde no trabalho (SSST n. 3), de 10/03/1994. A sua capacidade em provocar dados cromossômicos e à medula óssea já foram amplamente demonstrados em seres humanos e animais [16].

Experimentos de adsorção anteriores demonstraram eficiência satisfatória das organoargilas em relação ao carvão ativado nos tratamentos de águas contaminadas com os compostos orgânicos que evaporam em temperatura ambiente [17]. Esta característica indica que é viável utilizar em alguns processos de adsorção as organoargilas que são de baixo custo, comparados com o carvão ativado, que é um adsorvente de alta eficiência para compostos orgânicos voláteis

Neste estudo foi avaliado o uso de uma amostra de solo argiloso do Estado do Acre como material precursor na obtenção de organoargila a partir de tratamentos com hexadeciltrimetilamônio e sua aplicabilidade na adsorção de fenol e benzeno em fase líquida.

\section{MATERIAIS E MÉTODOS}

Utilizou-se uma argila natural de natureza esmectítica, proveniente do Estado do Acre, na sua forma bruta, peneirada (ABNT 200 mesh), sem modificação das propriedades químicas e com CTC de $60 \mathrm{cmol}_{\mathrm{c}} \mathrm{kg}^{-1}$ [18]. $\mathrm{O}$ reagente utilizado na modificação foi o brometo de hexadeciltrimetilamonio 99\% da Sigma Aldrich.

A organoargila foi obtida conforme os procedimentos iniciais empregados em estudo anterior, que consiste na preparação de uma suspensão aquosa da argila natural e adição de hexadeciltrimetilamonio (HDTMA) em concentrações equivalentes 0,05 a 5 vezes a capacidade de troca catiônica da argila precursora e agitação das suspensões por 7 dias em temperatura ambiente [4]. Foram adicionadas quantidades de HDTMA equivalentes a 1, 4 e 5 ao valor de CTC da argila precursora. Em seguida o material obtido foi submetido a várias lavagens com água deionizada até obtenção de soluções sobrenadantes transparentes, livres de espumas e com resultados negativos para cloreto, para posterior secagem em temperatura ambiente durante $24 \mathrm{~h}$.
Após a modificação, os sólidos obtidos foram nomeados HDTMA-arg 1, HDTMA-arg 4 e HDTMA-arg 5.

Os materiais (argila natural e organoargilas) foram submetidos às seguintes técnicas de caracterização:

- Difração de raios X (DRX) em um difratômetro Panalytical X'Pert Pro MPD com radiação $\mathrm{k}_{\alpha}$ do cobre $\quad(\lambda$ $=1,540598 \AA$ ) , passo de $0,03 \mathrm{em} 2 \theta$. As análises foram pelo método do pó e os difratogramas foram registrados a partir de programa adequado para análises preliminares, visando estimativa das posições de picos em baixos ângulos e programa com indicação mais precisa das posições que é específico para microanálises, a fim de comprovar as expansões esperadas para as argilas modificadas.

- Microscopia eletrônica de varredura (MEV): foram obtidas em um microscópio LEO-1430 equipado com o sistema de energia dispersiva de raios X (EDS). As condições de análises para imagens de elétrons secundários foram: corrente do feixe de elétrons de $90 \mu \mathrm{A}$, voltagem de aceleração de $10 \mathrm{kV}$ e distancia de trabalho $12-15 \mathrm{~mm}$. As amostras foram previamente metalizadas com uma fina camada de ouro e platina. Estas técnicas têm a finalidade de verificar se a HDTMA-arg 5 preservou em parte a estrutura da esmectita original e identificar a composição química das amostras.

- Espectroscopia na região vibracional do infravermelho com transformada de Fourier (FTIR), foi feita em um espectrofotômetro Perkin Elmer 1760 x FTIR na região de 4000 a $400 \mathrm{~cm}^{-1}$ em atmosfera autogerada, a fim de indicar as vibrações características dos materiais obtidos em cada etapa do processo de síntese e após tratamentos dos materiais, e com isso caracterizar a natureza química dos sítios ácidos superficiais.

Para os testes de adsorção foram utilizadas duas séries de 8 porções de aproximadamente $20 \mathrm{mg}$ da organoargila (HDTMA-arg 5) contidas em tubos de ensaio. Soluções de fenol e benzeno com concentrações iniciais no intervalo 2.5 a $15 \mathrm{mgL}^{-1}$ foram adicionadas em cada série de tubos de ensaios contendo a organoargila. As suspensões aquosas foram agitadas por $1 \mathrm{~h}$, colocadas em repouso por $24 \mathrm{~h}$ e centrifugadas por $15 \mathrm{~min}$ a $3000 \mathrm{rpm}$.

As concentrações do fenol e do benzeno foram medidas em espectrofotômetro 50 Probe Varian, com comprimento de onda $270 \mathrm{~nm}$ e $209 \mathrm{~nm}$ para fenol e benzeno, respectivamente. A capacidade de sorção (qe) do fenol e benzeno foi calculada por meio da equação A.

$$
q e\left(\mathrm{mg} \mathrm{g}^{-1}\right)=(C \mathrm{i}-C e) \times V / m
$$

na qual $C$ i e $C$ e são as concentrações de equilíbrio iniciais do fenol e do benzeno nas soluções $\left(\mathrm{mgL}^{-1}\right)$ e $V$ é o volume (L) das suspensões aquosas e $m$ é a massa do adsorvente em gramas $(\mathrm{g})$.

$\mathrm{Na}$ descrição dos processos de adsorção foram testados os modelos de isotermas de adsorção de Langmuir e Freundlich. A análise dos dados experimentais foi efetuada a partir de ajuste não linear usando o software Origin 7.0.

A espontaneidade dos processos de adsorção foi 
avaliada com base na energia livre de Gibbs calculada pela equação B.

$$
\Delta \mathrm{G}^{\mathrm{o}}=-\mathrm{RT} \ln K
$$

na qual $\mathrm{R}$ é a constante universal do gases $\left(8,314 \mathrm{~J} \mathrm{~mol}^{-1} \mathrm{~K}^{-1}\right)$, T é a temperatura absoluta (K) e $K$ é a constante aparente da reação de adsorção. $\mathrm{O}$ sinal de $\Delta \mathrm{G}^{\circ}$ indica se a reação é espontânea ou não, num sistema fechado a uma dada temperatura.

\section{RESULTADOS E DISCUSSÃO}

A partir dos resultados de DRX obtidos nas análises preliminares (a) e microanálises (b) da argila natural e organoargila (Fig. 1) foram feitas as seguintes observações: a amostra natural é classificada como argilomineral, com a presença da esmectita caracterizada pela distância interplanar de $1,47 \mathrm{~nm}$; a amostra tratada com menor quantidade do sal quaternário de amônio (HDTMA-arg 1) não atingiu as características esperadas de intercalação mínima com o

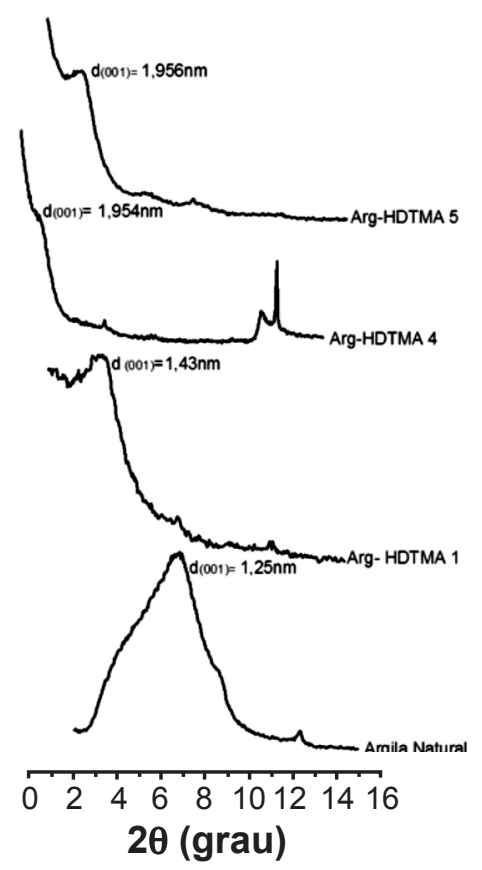

Figura 1: Difratogramas de raios $\mathrm{X}$ das amostras: argila natural, Arg-HDTMA 1, Arg-HDTMA 4 e Arg-HDTMA 5.

[Figure 1: X-ray diffraction patterns of samples: natural clay, ArgHDTMA 1, Arg-HDTMA 4 and Arg-HDTMA 5.] surfactante utilizado enquanto que as amostras tratadas com quantidades superiores do surfactante (HDTMA-arg $4 \mathrm{e}$ HDTMA-arg 5) apresentaram uma mudança nos padrões de difração com expansões muito próximas. A microanálise (b) mostra de forma mais detalhada as mudanças nos valores de $2 \theta$ e os respectivos valores de $d_{001}$ da argila natural e após tratamento com HDTMA em quantidade cinco vezes ao valor de CTC da argila. Observa-se que a reflexão da argila sem tratamento está situada entre $4,425^{\circ}$ e $8,8^{\circ}(2 \theta)$ e que a intensidade máxima da reflexão corresponde ao valor de $\mathrm{d}_{001}=1,47 \mathrm{~nm}$. Após a intercalação o pico em 1,47 nm da esmectita expandiu para $1,91 \mathrm{~nm}$ e foi deslocado em direção a ângulos mais baixos, ficando entre $4,61^{\circ} \mathrm{e} 4,1^{\circ}$, esta mudança corresponde a uma expansão $78,81 \%$ no valor de $d_{001}$.

Os resultados da análise de espectroscopia de raios $\mathrm{X}$ por energia dispersiva (EDS) e de microscopia eletrônica de varredura (MEV) são mostrados respectivamente na Tabela I e na Fig. 2.

Nas análises de EDS realizadas a partir varreduras nas áreas A e B indicadas na Fig. 2 observou-se que a amostra natural sem tratamento é composta basicamente de Si e Al (Tabela I), o que é característico de argilas esmectíticas, além da presença de $\mathrm{C}, \mathrm{Na}, \mathrm{Mg}, \mathrm{K}$ e Fe. $\mathrm{Na}$ amostra HDTMA-arg 5 foi possível identificar os mesmos elementos da argila natural, entretanto observouse um aumento considerável na concentração do carbono e decréscimo na concentração de $\mathrm{Na}$ e Al. Esta característica indica que a organofuncionalização propiciou ataque tanto das folhas tetraédricas como octaédricas da argila original. As micrografias (Fig. 2) não mostram mudanças muito significativas entre os aspectos morfológicos das amostras de argila sem tratamento com HDTMA. (Fig. 3, área A), pois ambas apresentam uma distribuição não homogênea dos grãos, os quais possuem tamanhos diferentes, formando aglomerados de forma compactada e irregular.

A intercalação do cátion HDTMA foi avaliada pela comparação dos espectros FT-IR da amostra original e da amostra modificada quimicamente (Fig. 3). A argila natural foi caracterizada como montmorilonita, apresentando bandas na região de $3630 \mathrm{~cm}^{-1}$, que corresponde ao estiramento da ligação $\mathrm{O}-\mathrm{H}$, em 3450 e $1646 \mathrm{~cm}^{-1}$, que corresponde ao grupo $(-\mathrm{OH})$ hidratado, uma banda larga em $1003 \mathrm{~cm}^{-1}$ referente à vibração de alongamento do Si$\mathrm{O}$, e em $798 \mathrm{~cm}^{-1}$ que é referente ao grupo $\mathrm{OH}$ ligado ao $\mathrm{Al} / \mathrm{Mg}$. Nos espectros da argila modificada (HDTMA-arg 5), ocorreu o aparecimento de bandas que caracterizam o estiramento assimétrico e simétrico das ligações $\mathrm{C}-\mathrm{H}$ dos

Tabela I - Análise elementar (\%) das amostras: Argila-natural (a) e HDTMA-arg 5 (b). [Table I - Elemental analysis (\%) of samples: natural clay and HDTMA-arg 5.]

\begin{tabular}{ccccccccc}
\hline Elementos & $\mathrm{C}$ & $\mathrm{O}$ & $\mathrm{Na}$ & $\mathrm{Mg}$ & $\mathrm{Al}$ & $\mathrm{Si}$ & $\mathrm{K}$ & $\mathrm{Fe}$ \\
\hline Argila natural & 0,30 & 46,07 & 0,77 & 0,97 & 12,35 & 30,33 & 1,68 & 4,78 \\
HDTMA-arg 5 & 19,70 & 40,71 & 0,15 & 0,73 & 3,31 & 26,90 & 1,21 & 3,73 \\
\hline
\end{tabular}




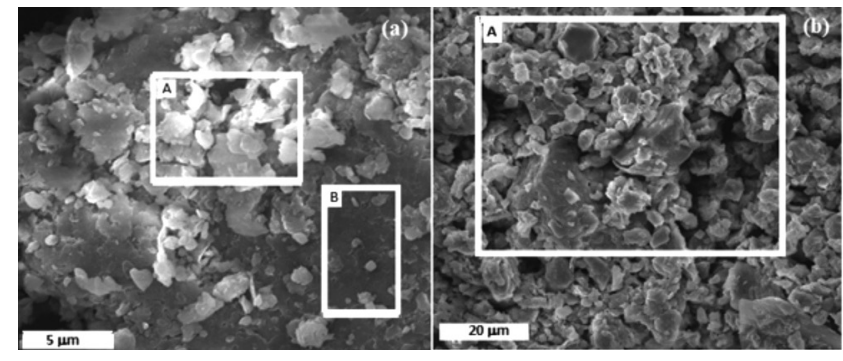

Figura 2: Micrografias obtidas por microscopia eletrônica de varredura das amostras: argila natural (a) e HDTMA-arg 5 (b). [Figure 2: Scanning electron microscopy micrographs of samples: natural clay (a) and HDTMA-arg 5 (b).]

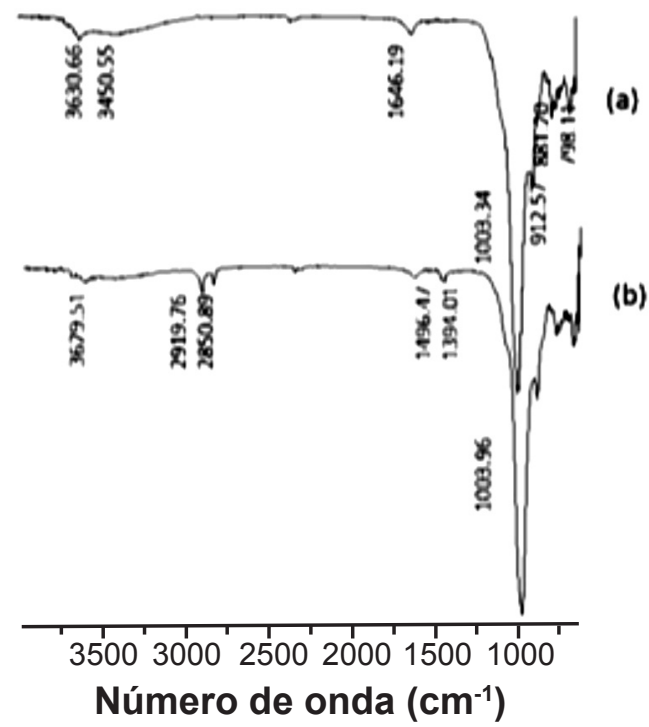

Figura 3: Espectros de infravermelho das amostras: argila natural (a) e HDTMA-arg 5 (b).

[Figure 3: Infrared spectra of the samples: natural clay (a) and HDTMA-arg 5 (b).]

grupos $\mathrm{CH}_{3}$ e $\mathrm{CH}_{2}$ na região de 2919 e $2850 \mathrm{~cm}^{-1}$, na região de $149611394 \mathrm{~cm}^{-1}$, também foram encontradas bandas de absorção que correspondem ao estiramento da ligação N-H proveniente do cátion hexadeciltrimetilamônio, indicando que este cátion foi intercalado nos espaços interlamelares da argila natural.

\section{Dados de adsorção}

A partir dos modelos de isotermas selecionados na descrição do equilíbrio nas interfaces sólidos/soluções, representadas por HDTMA- arg 5 e soluções de fenol e benzeno (Fig. 4 e Tabela II) foi verificado com base no coeficiente $\mathrm{R}^{2}$ e $\mathrm{n}_{\mathrm{F}}$ que os dois modelos de isotermas ajustaram de forma satisfatória os dados experimentais de adsorção e que esses processos são favoráveis e heterogêneos $\left(\mathrm{n}_{\mathrm{F}}>1\right)$. Foram observados nos dois processos de adsorção: valores das constantes $\mathrm{K}_{\mathrm{L}}$ bem inferiores a unidade, indicando baixa afinidade química entre os adsorvatos e adsorvente; valores
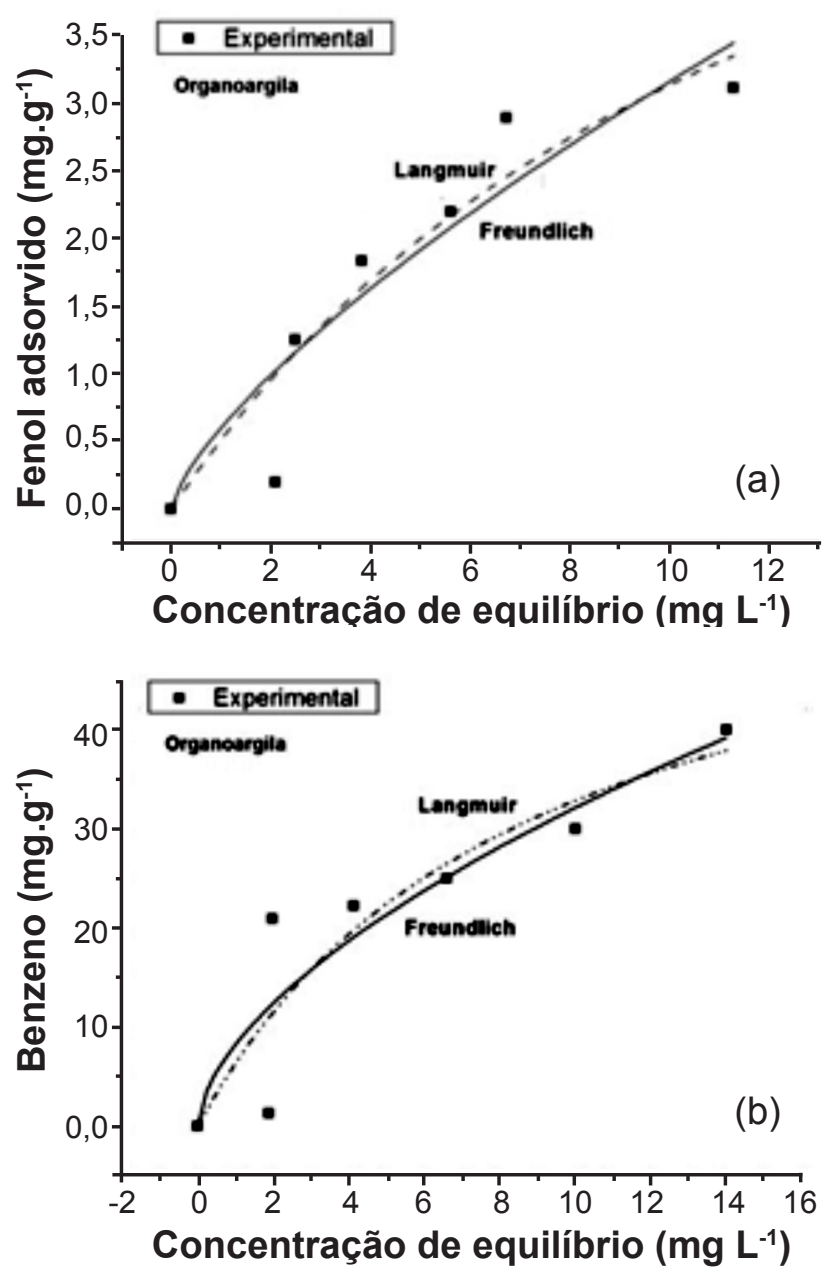

Figura 4: Isotermas de adsorção do fenol e benzeno em HDTMA$\arg 5$.

[Figure 4: Adsorption isotherms of phenol and benzene at HDTMAarg 5.]

Tabela II - Parâmetros das isotermas ajustados aos modelos de Langmuir e Freundlich.

[Table II - Isotherm parameters fitted to Langmuir and Freundlich.]

\begin{tabular}{lccccccc}
\hline $\begin{array}{l}\text { Amostra - } \\
\text { HDTMA-arg } 5\end{array}$ & \multicolumn{2}{l}{ Langmuir } & \multicolumn{5}{c}{ Freundlich } \\
\hline Parâmetros & $\mathrm{q}_{\max }$ & $\mathrm{K}_{\mathrm{L}} \mathrm{R}^{2}$ & $\mathrm{~K}_{\mathrm{F}}$ & $\mathrm{n}_{\mathrm{F}}$ & $\mathrm{R}^{2}$ & $\Delta \mathrm{G}^{\mathrm{o}}$ \\
\hline Fenol & 4,44 & 0,07 & 0,89 & 0,48 & 1,01 & 0,87 & $-16,85$ \\
Benzeno & 35,8 & 0,11 & 0,83 & 4,95 & 1,18 & 0,84 & $-18,38$ \\
\hline
\end{tabular}

significativos para os parâmetros indicadores de quantidades adsorvidas $\left(\mathrm{q}_{\max }\right.$ e $\left.\mathrm{K}_{\mathrm{F}}\right)$, que sugerem eficiência de adsorção em fase líquida muito maior para o benzeno do que para o fenol; baixos valores absolutos de $\Delta \mathrm{G}^{0}$ que são indicativos de predomínio de interação física do adsorvato com o adsorvente. A quantidade máxima adsorvida de benzeno obtida na organoargila neste estudo encontra-se no intervalo citado para organominerais, situado entre aproximadamente 
20 a 40 mg. $\mathrm{g}^{-1}$ [16] mas inferior as obtidas em carvão ativado que é considerado um dos adsorventes mais eficientes na remoção de benzeno em fase líquida e apresentando $\mathrm{q}_{\max }$ de benzeno $=110$ a $270 \mathrm{mg} \cdot \mathrm{g}^{-1}$ [13]. No caso da adsorção do fenol o valor da quantidade adsorvida encontra-se tanto no intervalo publicado para organoargila [9] como em carvão ativado [10].

\section{CONCLUSÕES}

As seguintes conclusões foram obtidas no estudo sobre a intercalação de esmectita do estado Acre com o cátion orgânico HDTMA em concentrações compreendidas entre 1 a 5 vezes ao valor de CTC da argila original e a aplicabilidade da organoargila HDTMA-arg 5 como adsorvente de fenol e benzeno em fase líquida: Os processos de intercalações em concentrações 4 e 5 vezes ao valor de CTC da argila original resultaram na formação de organoargilas com valores de $\mathrm{d}_{001}$ próximos de 1,91 nm; A formação da organoargila HDTMA-arg 5 propiciou alterações morfológicas e químicas em relação à argila precursora. Esta característica foi enfatizada pelo grande decréscimo nas concentrações dos elementos das posições interlamelares, como $\mathrm{Na}, \mathrm{K}, \mathrm{Ca}$ e $\mathrm{Mg}$, além das concentrações de $\mathrm{Al}$ e $\mathrm{Fe}$ das folhas octaédricas. Os processos de adsorção do benzeno e fenol em fase líquida na organoargila HDTMA-arg 5 foram considerados favoráveis, espontâneos e com interações físicas.

\section{AGRADECIMENTOS}

Ao $\mathrm{CNPq}$ pelas bolsas de doutorado à primeira autora e de pesquisa à segunda. Os autores agradecem ainda ao Prof. Dr. M. L. da Costa pela coleta e concessão da amostra (argila do estado do Acre), ao Prof. Dr. R. S. Angélica pelo grande apoio dado durante as análises de difração de raios $\mathrm{X}$ e pela grande dedicação como coordenador do PPGG/ UFPA.

\section{REFERÊNCIAS}

[1] L. Zampori, P. G. Stampino, G. Dotelli, D. Botta, I. N. Sora, M. Setti, Appl. Clay Sci. 41 (2008) 149.

[2] A. C. V. Coelho, P. S. Santos, H. S. Santos, Quim. Nova 30 (2007) 1282.

[3] M. Janek, G. Lagaly, Colloid Polym. Sci. 281 (2003) 293.

[4] S. Y. Lee, W. J. Cho, P. S. Hahn, M. Lee, Y. B. Lee, K. J. Kim, Appl. Clay Sci. 30 (2005) 174.

[5] V. N. Moraru, Appl. Clay Sci. 19 (2001) 11.

[6] E. J. Sullivan, J. W. Carey, R. S. Bowman, J. Colloid Interface Sci. 206 (1998) 369.

[7] S. Yariv, H. Cross, Organo-clay complexes and interactions, Ed. Marcel Dekker, New York, EUA (2002) 688.

[8] F. R.V. Díaz, Key Eng. Mater. 189-191 (2001) 203.

[9] A. A. Silva, F. R. V. Diaz, G. S. V. Martins, M. G. F. Rodrigues, Cerâmica 53, 328 (2007) 417.

[10] R. D. Vidic, M. T. Suidan, R. C. Brenner, Environ. Sci. Technol. 27 (1993) 2079.

[11] M. C. Ncibi, S. Altenor, B. Mahjoub, M. Seffen, S. Gaspard, 12th Int. Water Technology Conf., Alexandria, Egito (2008).

[12] G. Rytwo, Y. Kohavi, I. Botnick, Y. Gonen, Appl. Clay Sci. 36 (2007) 182.

[13] M. A. L. Ródenas, D. C. Amorós, A. L. Solano, Carbon 43 (2005) 1758.

[14] J. W. Choi, N. C. Choi, S. J. Lee, D. J. Kim, J. Colloid Interface Sci. 314 (2007) 367.

[15] C. Liang, Y. J. Chen, J. Hazardous Mater. 182 (2010) 544.

[16] E. R. L. Tiburtius, P. P. Zamora, Quim. Nova 27 (2004) 441.

[17] S. M. Kho, J. B. Dixon, Appl. Clay Sci. 18 (2001) 111. [18] S. S. Santos, V. P. Lemos, M. L. Costa, R. M. A. Rodrigues, O. V. Monteiro Junior, K. S. Oliveira, XV Int. Clay Conf., Rio de Janeiro, Brasil (2013) 611.

(Rec. 13/11/2013, Ac. 10/10/2014) 